Целью исследования является повышение эффективности процесса управления инцидентами в информационной системе предприятия. В статье проведен анализ работ, посвященных совершенствованию процесса управления инцидентами. Обоснована целесообразность применения ряда ключевых метрик, позволяющих оценить степень достижения показателями процесса их целевых значений, т. е. оценить качество управления иниидентами: скорости решения иниидента, степени удовлетворенности пользователей сервиса и доступности каналов обработки обращений пользователей.

Проведен сравнительный анализ существуюшей модели процесса управления инцидентов и предлагаемой модели. Предлагаемая модель, включающая дополнительную линию поддержки, позволяет существенно улучшить ключевые показатели процесса обработки и разрешения инцидентов.

Научная новизна разработанных предложений заключается в комплексном использовании совокупности процессных, технологических и сервисных метрик, обеспечивающем построение более эффективной модели управления процессом обработки инцидентов.

Материалы и методы. Теоретическую основу исследования составляет анализ рекомендаций по применению метрик в соответствии: с методологией управления информационными технологиями СОВIT, рекомендациями построения процесса управления инцидентами на основе библиотеки инфраструктуры информационных технологий ITIL, а также результатов научно-исследовательских работ российских и зарубежных ученых и публикаций ведущих организаций в области управления инцидентами в информационных системах предприятий. Проведен анализ метрик процессов управления инцидентами. Использованы математические методы количественного из- мерения ключевых метрик. Проведен анализ статистических данных, поступающих в службу технической поддержки проиессов управления инцидентами.

Результаты. Обосновано применение ключевых метрик, с помошью которых решается задача оперативного реагирования на инциденты, их последующей обработки и разрешения в условиях обеспечения гарантированной доступности каналов обработки обрашений. Разработана трехуровневая модель управления иниидентами, которая позволила более эффективно решать задачу управления процессами их обработки на основе комплексного применения ключевых метрик.

Заключение. В ходе проведенного исследования выявлены недостатки существующей модели процесса управления инцидентами. Проведен анализ метрик, используемых в существуюших моделях процесса управления инциентами. Обоснован выбор набора релевантных метрик, комплексное применение которых позволило разработать более эффективную модель управления инцидентами, отвечающую как требованиям потребителей услуг, так и требованиям, предъявляемым к функционированию информационной системы. Разработанная модель обеспечивает повышение качества обработки инцидентов (скорости, полноты, надежности).

Отличительной особенностью разработанной модели является использование объективных количественных характеристик, полученных на основе релевантных метрик процесса управления инцидентами, которые позволили обосновать предложения по совершенствованию существующей модели управления инцидентами в информационной системе предприятия.

Ключевые слова: модель управления инцидентами, ключевые (релевантные) метрики.

\title{
Development of the Incident Management Model in an Enterprise Information System Based on a Three-Tier Architecture Using Key (Relevant) Metrics
}

The aim of the study is to increase the efficiency of the incident management process in an enterprise information system. The article analyzes the work on improving the incident management process. The expediency of applying a number of key metrics is substantiated, which makes it possible to assess the degree to which the process indicators achieved their target values, that is, assess the quality of the incident management: the speed of solving the incident, the degree of satisfaction of service users and the availability of channels for processing user requests. A comparative analysis of the existing model of the incidents management process and the proposed model is performed. The proposed model, which includes an additional support line, can significantly improve key indicators of incident handling and resolution process. The scientific novelty of the developed proposals lies in the integrated use of a combination of process, technological and service metrics, which provides the construction of a more effective model of incident management.

Materials and methods. The theoretical basis of the study is the analysis of recommendations for the use of metrics in accordance with: the management methodology of the COBIT information technology, recommendations for building an incident management 
process based on the ITIL library of information technology infrastructure, as well as the results of scientific research by Russian and foreign scientists and publications of leading organizations in the field of management incidents in enterprise information systems. The analysis of incident management process metrics is carried out. The mathematical methods of quantitative measurement of key metrics are used. The analysis of statistical data received by the technical support service for incident management processes was carried out.

Results. The use of key metrics is justified, with the help of which the task of promptly responding to incidents, their subsequent processing and resolution is solved in conditions of ensuring guaranteed access to channels for processing calls. A three-tier incidents' management model was developed, which made it possible to more effectively solve the problem of managing their processing based on the integrated use of key metrics.

\section{Введение}

Эффективность деятельности компании напрямую зависит от слаженной (гармоничной) работы руководства бизнеса и ИТ-специалистов. Сложности в построении конструктивного диалога между руководством компании и ее ИТ-департаментом, приводят к росту издержек, выполнению достаточно большого объема рутинной работы. С целью ликвидации разрыва между руководством и ИТ-департаментом применяют различные подходы, например на основе методологии COBIT, которая вводит ряд показателей для оценки эффективности реализации системы управления информационными технологиями (ИТ) в компании $[1,2]$. В ИТ-департаменте функционирует служба технической поддержки, которая решает задачи реагирования на различные инциденты (в статье не рассматриваются инциденты информационной безопасности), связанные с функционированием информационной системы компании. Под инцидентом понимается незапланированное прерывание или снижение качества ИТ-услуги [3, 4]. Инцидент может вызвать приостановку процесса предоставления сервиса или снижение качества его работы, в результате чего поступают массовые обращения от пользователей сервиса. Таким образом, под инцидентом можно понимать любое событие, не являющееся частью нормального (штатного) функционирования системы/сервиса/ процесса [5].

Основной задачей службы технической поддержки, которая является составной частью ИТ-департамента является обеспечение поддержания непрерывности бизнеса путем управления жизненным циклом инцидентов. Скорость реакции на инцидент и сроки решения инцидентов, регистрируемых в службе технической поддержки, должны соответствовать значениям, принятым в Соглашении об уровне предоставления услуг (SLA), основном документе, регламентирующем взаимодействие службы ИТ и заказчика, в роли которого может выступать как бизнес-руководство, так и конечный пользователь услуги. Основная цель процесса управле-
Conclusion. The study revealed the shortcomings of the existing model of the incident management process. The analysis of metrics used in existing models of the incident management process is carried out. The choice of a set of relevant metrics is substantiated, the complex application of which allowed us to develop a more effective incident management model that meets both the requirements of service consumers and the requirements for the operation of an information system. The developed model provides improved quality of incident processing (speed, completeness, reliability).

A distinctive feature of the developed model is the use of objective quantitative characteristics obtained on the basis of relevant metrics of the incident management process, which made it possible to substantiate proposals for improving the existing incident management model in the enterprise information system.

Keywords: incident management model, key (relevant) metrics.

ния инцидентами - скорейшее восстановление услуги для клиентов [5].

Для повышения эффективности процесса управления инцидентами используются многочисленные инструменты, как правило, это различные информационно-аналитические системы, такие как, HP ServiceDesk, IBM ServiceDesk, HelpDesk, Jira, ВPM-Online. Подобные системы являются дорогостоящими, и их достаточно сложно адаптировать к нуждам конкретной службы технической поддержки, учитывая всю специфику ее работы.

Проведенный анализ показал, что существующие модели управления инцидентами в условиях функционирования распределенных информационных систем компании с большим количеством пользователей, в том числе удаленных, не в полной мере соответствуют предъявляемым требованиям, и не всегда обеспечивают необходимый уровень предоставления сервисов.

Научная новизна работы определяется обоснованием необходимости построения трехуровневой модели обработки инцидентов с использованием базы знаний, обеспечивающей поддержание процесса в актуальном состоянии и своевременное реагирование на поступающие запросы. Предложен подход, обеспечивающий повышение доступности каналов обработки обращений за счет определения их минимально необходимого количества. Использование релевантных метрик для объективной оценки степени достижения показателями процесса управления инцидентами их целевых значений позволило повысить эффективность последнего.

\section{Анализ существующих моделей процесса управления инцидентами}

Проблематике повышения эффективности процесса управления инцидентами посвящено достаточно большое количество исследований.

Так, в работе [6], предложен комплекс моделей, алгоритмов и программных средств на базе библиотеки ITIL, обеспечивающих повышение эффективности обработки запросов, поступаю- 
щих в службу технической поддержки Интернет-провайдера за счет рационального распределения персонала по работам и приоритезации запросов пользователей.

В работе [7] анализируются вопросы повышения качества разрешения инцидентов. В ней представлены математические модели и методы управления инцидентами, в частности математическая модель, описывающая зависимость количества обращений от числа обслуживаемых рабочих мест.

В работе [8] для оценки и контроля качества ИТ-услуг предложено рассматривать сервисные показатели эффективности, такие как процент пропущенных звонков за период, процент решенных инцидентов за период, среднее количество заявок на второй линии поддержки за период и среднее количество заявок за период на одном сотруднике второй линии поддержки.

Анализ результатов исследований в области управления инцидентами в информационной системе предприятия показал, что существующие модели процесса управления инцидентами не в полной мере отвечают предъявляемым требованиям.

На рис. 1 представлена структурная схема типовой модели управления инцидентами $[9,10]$.

В основе модели лежит двухуровневая система обработки инцидентов, включающая 1-ю линию поддержки, на которой осуществляется регистрация инцидента, классификация возникшей проблемы и реализация этапа начальной поддержки. Если в ходе предоставления

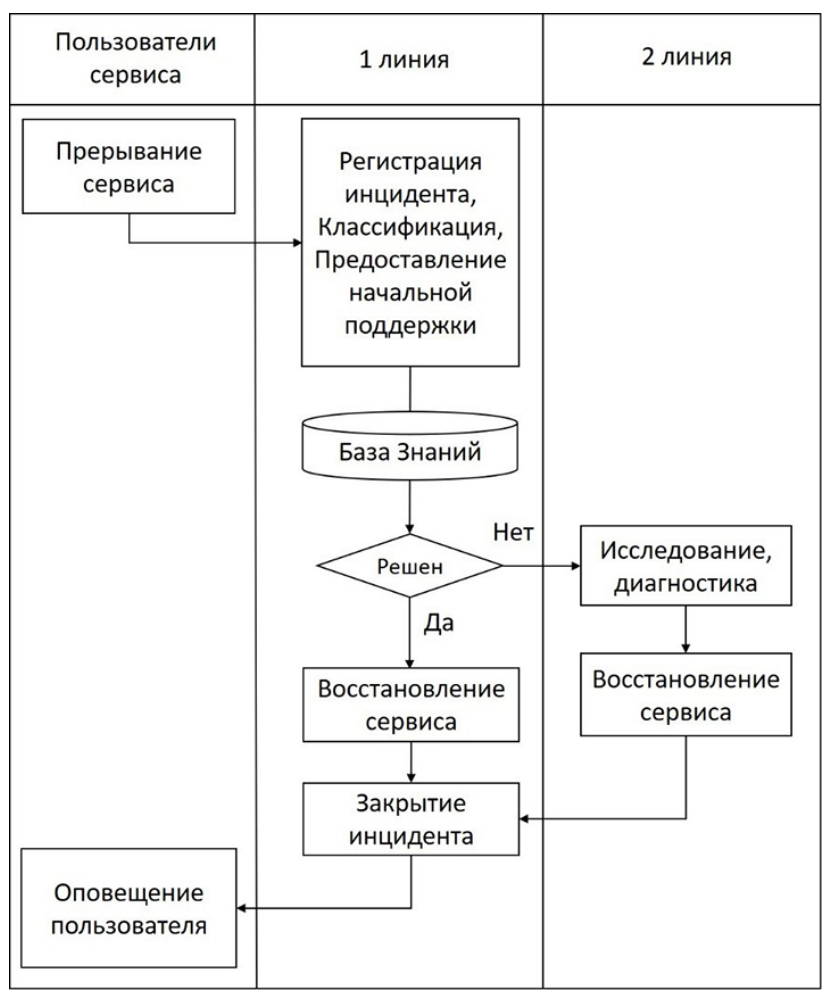

Рис. 1 Существующая модель процесса управления инцидентами начальной поддержки проблема устраняется, то инцидент закрывается. При невозможности решить инцидент в рамках первой линии инцидент направляется на вторую линию поддержки. Инженеры второй линии более глубоко исследуют возникшую проблему, диагностируют неисправности и направляют результат на первую линию для закрытия. Если проблему не удалось решить на второй линии технической поддержки, то проблема направляется на более глубокое исследование и диагностику, а первая линия информирует об этом пользователя [11].

Таким образом, в модели управления инцидентами на выходе генерируется сообщение пользователю о восстановлении сервиса, сохраняется запись об инциденте и запись о возникшей проблеме. При этом в базе знаний накапливается статистика: количество открытых инцидентов, отсортированных по приоритету, по прошедшему времени, по рабочим группам; количество инцидентов, разрешенных на каждой линии поддержки; среднее время решения инцидента в рабочей группе; среднее время восстановления сервиса, процент инцидентов, peшенных в рамках крайнего срока и др. [12, 13].

При анализе существующей модели управления инцидентами выявлены следующие недостатки:

- образование очередей и низкая доступность каналов обработки обращений при массовых сбоях;

- на первой линии регистрация и классификация обращений занимает значительное время, при этом практически не остается время для консультирования и наиболее важным недостатком является не структурированность и неактуальность базы знаний, что приводит к невозможности ее использования в ходе разрешения инцидента;

- передача инцидента на 2-й уровень поддержки зачастую осуществляется до окончания его разрешения на 1-ом уровне. Проблема маршрутизации большинства инцидентов на вторую линию возникает из-за ограниченного времени на поиск ответа в существующей базе знаний. Указанная проблема существенно влияет на скорость разрешения инцидента;

- превышение установленных сроков обработки инцидента в соответствии с принятым на предприятии Соглашением об уровне оказания услуг (англ. Service Level Agreement, SLA). Каждый инцидент, зарегистрированный в информационной системе управления инцидентами, имеет свои сроки выполнения. Указанные в SLA уровни качества предоставления услуг, сроки восстановления и время доступности предоставляемых услуг должны соблюдаться $[14,15]$.

С учетом сказанного, сформулирована задача разработки модели процесса управления инци- 
дентами, которая должна обеспечить повышение его эффективности. С этой целью обоснована необходимость использования набора релевантных метрик, таких как: скорость устранения инцидентов; удовлетворенность пользователей качеством ИТ-поддержки; уровень доступности каналов обработки обращений. Для обеспечения более гибкого процесса обработки инцидентов разработанная модель процесса управления инцидентами включает дополнительную линию поддержки, обеспечивающую обработку сложных инцидентов, мониторинг ошибок, сбоев, имеющих критический и блокирующий приоритеты, следствием чего могут быть крупные финансовые риски, падение уровня сервиса, доступности, а также работоспособности сервиса.

Реализация предложенного подхода позволила существенно повысить значения указанных показателей (скорости решения инцидентов на $43,4 \%$, степени удовлетворенности пользователей - на $81 \%$, доступности каналов обработки обращений - на 45,4\%).

\section{2. Разработка трехуровневой модели управления инцидентами}

Для повышения эффективности процесса управления инцидентами в соответствии с методологией COBIT и библиотекой ITIL применяются различные метрики, которые должны соответствовать принципам SMART (то есть быть конкретными, измеримыми, достижимыми, актуальными и привязанными к определенному интервалу времени) [16]. Метрики, представляющие собой количественные меры степени достижения процессами своих целей, позволяют оценить качество процесса управления инцидентами, возможности достигать запланированных результатов и в итоге оценить их эффективность.

В работах $[6,7,8]$ рассмотрена классификация метрик с привязкой к компонентам информационной системы. Анализ метрик, представленных в методологии СОВІТ и библиотеке ITIL, показал, что множество существующих метрик соответствуют следующим основным классам объектов информационной системы: инфраструктура, процессы и сервисы. В соответствии с указанной классификацией, выделяют технологические (метрики компонентов и приложений, такие как производительность, доступность и др.), процессные (отражают эффективность функционирования внутренних процессов ИТ) и сервисные метрики (отражают качество предоставления услуги, значения параметров согласованных в SLA).

Для построения модели процесса управления инцидентами и определения наиболее значимых (релевантных) метрик рассматриваемого процесса принят во внимание тот факт, что целью процесса управления инцидентами является гарантированное реактивное устранение последних, важным показателем которого является скорость реакции на инцидент [16,17]. Она позволяет дать количественную оценку затрат времени, потраченного на первой и второй линии поддержки при регистрации, классификации, консультировании, маршрутизации, детальном разборе инцидента, обращении к базе знаний и др.

Кроме того, одним из важных показателей является своевременность обработки обращений от пользователей (это особенно важно для конечного заказчика ИТ-услуги). В качестве показателя качества услуги со стороны пользователя используется метрика - оценка удовлетворенности пользователей качеством ИТ-поддержки [18]. Данная метрика позволяет количественно оценить, насколько удобной и быстрой была помощь, оказанная конечному пользователю заказчику услуги.

В условиях достаточно большого потока обращений от пользователей с учетом возникающих сбоев необходимо обеспечить гарантированную доступность каналов обработки обращений, либо минимизировать (снизить) вероятности отказа в обслуживании, используя одноименную метрику.

Скорость реакции на инцидент может быть рассчитана следующим образом. Свойство процесса управления инцидентами таково, что свой вклад в скорость решения инцидента вносят все участники процесса его обработки. Для измерения данной метрики с учетом линий поддержки используется формула (1):

$$
K_{1}=\sum_{i}\left(W_{i j} \cdot R_{i j}\right) / \sum_{i} W_{i j},
$$

где: $R_{i j}-$ рейтинг обработки $i$-того инцидента в $j$-той линии, определяемый формулой (2):

$$
R_{i j}=\left\{\begin{array}{l}
1, \text { если инцидент обработан в срок, } \\
1-\frac{t_{i j}}{T_{i}}, \text { если инцидент просрочен и } t_{i j} \leq T_{i} ;
\end{array}\right.
$$

$W_{i j}-$ вес $i$-того инцидента для $j$-той линии, определяемый по формуле (3):

$$
W_{i j}=\left\{\begin{array}{l}
1, \text { если } t_{i j} \leq T_{i} \\
\left(\frac{t_{i j}}{T_{i}}\right)^{n}, \text { если } t_{i j}>T_{i} .
\end{array}\right.
$$

В формулах (2), (3) $t_{i j}$ - время обработки $i$-ого инцидента в $j$-ой линии, $T_{i}-$ максимальное время обработки $i$-ого инцидента, $n$ - натуральный параметр алгоритма, обычно равный 1.

Показатель метрики $K_{1}$ рассчитывается следующим образом:

- если инцидент обработан своевременно, $R_{i j}$ и $W_{i j}$ равны 1 ;

- если инцидент просрочен, причем $j$-ая линия обрабатывала его дольше, чем полный срок 
обработки $T_{i}$, рейтинг $R_{i j}=0$, вес $W_{i j}$ больше 1 пропорционально времени обработки в линии;

- если инцидент просрочен, но $j$-ая линия обрабатывала его, например, в течение половины срока, вес $W_{i j}=1$, рейтинг $R_{i j}=0,5$;

- если какая-то линия обрабатывала просроченный инцидент в течение короткого времени и, следовательно, вряд ли существенно повлияла на его просрочку, рейтинг $R_{i j}$ будет близок к 1 , то есть снижение значения метрики $K_{1}$ для этой линии будет весьма незначительным [19].

Для измерения удовлетворенности пользователей качеством ИТ-поддержки проводится оценка удовлетворенности непосредственно перед закрытием решенного инцидента, как правило, обеспечивается средствами веб-интерфейса системы автоматизации ITSM-процессов или по телефону - пользователя просят оценить, насколько он удовлетворен оказанной ему поддержкой по некоторой балльной шкале.

Численную оценку ответов пользователей (полученных как при закрытии их инцидентов, так и в результате проведения опроса) по произвольной целочисленной шкале удобно представить следующим образом, с помощью формулы (4):

$$
K_{2}=\frac{M-M_{\min }}{M_{\text {max }}-M_{\text {min }}}
$$

где $M-$ средний балл по ответам клиентов, $M_{\max }$ и $M_{\min }$ - минимальный и максимальный баллы по шкале оценок (для 5-ти балльной шкалы $M_{\max }=5$ и $\left.M_{\min }=1\right)$.

Для определения показателя метрики доступности каналов обработки обращений используется подход, рассмотренный в работе [20]. Процесс обращения пользователей в службу технической поддержки может быть представлен моделью массового обслуживания, позволяющей определить минимальное количество каналов обработки обращений, при котором вероятность отказа в обслуживании не превышает заданного значения. Количественная оценка вероятности отказа в обслуживании определяется по формуле (5):

$$
P_{\text {отв. }}=\frac{\rho^{i}}{i !} \cdot p_{0} ; i=1,2, \ldots n,
$$

где $P_{\text {отк. }}-$ вероятность отказа в обслуживании обращения, $\rho^{i}-$ коэффициент загрузки каналов приёма обращений, определяет среднее число поступающих обращений, приходящих за среднее время обслуживания одного обрашения, $i-$ количество обращений, $p_{0}-$ вероятность отказа в обслуживании, когда система находится в состояниях $S_{0}, S_{1}, \ldots, S_{i}$. Вероятность $p_{0}$ может быть рассчитана по формуле [7]:

$$
p_{0}=\left(1+\rho+\frac{\rho^{2}}{2 !}+\ldots+\frac{\rho^{i}}{i !}\right)^{-1} ; i=1,2, \ldots n
$$

Проведенный анализ недостатков существующей модели позволил сформулировать требо- вания к построению трехуровневой модели процесса управления инцидентами.

Модель процесса управления инцидентами должна отвечать следующим требованиям:

- обеспечение доступности каналов обработки обращений, исключающей их потерю;

- непрерывное поддержание базы знаний в актуальном состоянии;

- передача сложного инцидента для последующей обработки на дополнительную линию поддержки;

- исключение превышения установленных сроков обработки инцидента.

Разработанная модель представлена на рис.2.

В архитектуру модели для разгрузки специалистов 1-й и 2-й линий поддержки введена 3-я линия поддержки, обеспечивающая решение сложных инцидентов, требующих для этого дополнительных ресурсов.

Входными данными в модели являются обращения пользователей о прерываниях сервиса и сбоях. Обращения поступают на первую линию поддержки, которая осуществляет прием, регистрацию, классификацию, приоритизацию инцидентов и первичную консультацию. Каналы, по которым поступают обращения на первую линию: телефон, голосовое сообщение, электронная почта, социальные сети, мессенджеры, формы обратной связи через сайт, либо через мобильное приложение.

Линии поддержки используют единую базу знаний, которая обновляется в режиме реального времени. При отсутствии решения в единой базе знаний и невозможности оказания первичной консультации первая линия марш-

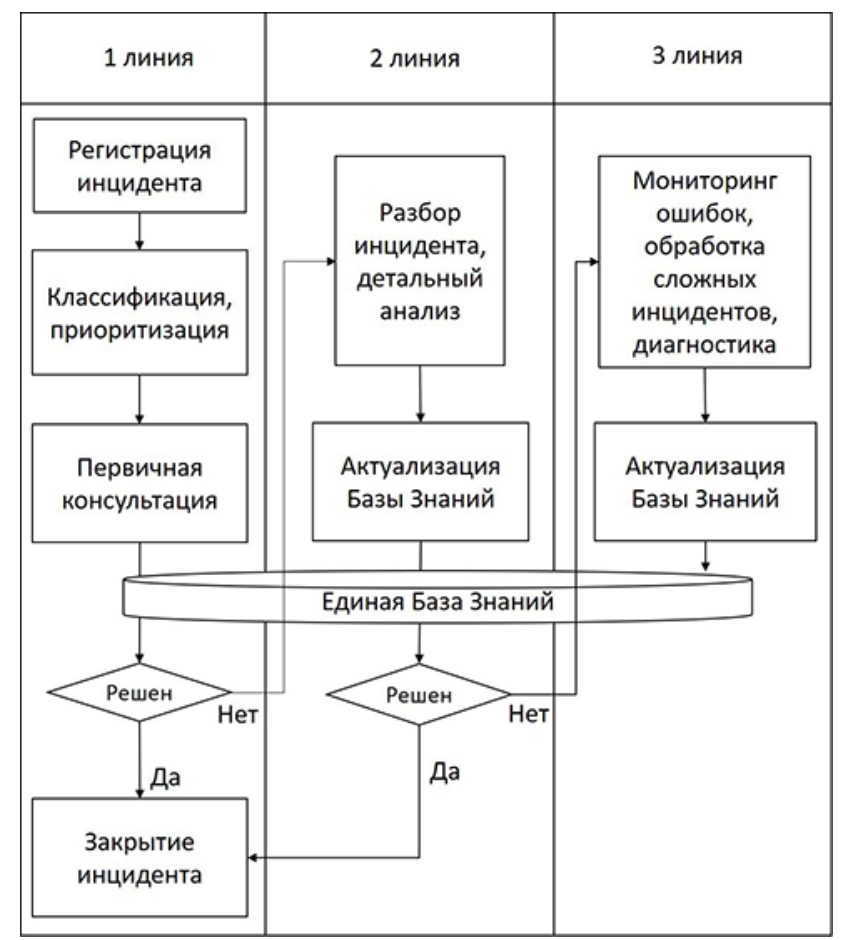

Рис. 2. Разработанная модель процесса управления инцидентами 
рутизирует инциденты на вторую линию поддержки.

На второй линии поддержки осуществляется решение инцидентов и их детальный анализ. Новые решения, полученные в ходе анализа инцидента, добавляются в единую базу знаний и являются доступными всем линиям поддержки для последующих консультаций по подобным инцидентам. Если инцидент не удается решить на уровне второй линии поддержки, он маршрутизируется на третью линию поддержки.

На третьей линии поддержки, осуществляется мониторинг ошибок, обработка сложных инцидентов, сбоев, имеющих критический и блокирующий приоритеты, которые могут повлечь за собой крупные финансовые риски, падение уровня сервиса, доступность, работоспособность сервиса.

Рассмотренные выше метрики позволили получить объективные сравнительные оценки показателей эффективности процесса обработки инцидента на всех этапах его жизненного цикла с использованием существующей и разработанной модели. Результаты проведенных оценок представлены в разделе 3.

\section{Сравнительный анализ показателей метрик существующей и разработанной модели}

Сравнительный анализ проведен с использованием ключевых метрик: $K_{l}-$ скорость реакции на инцидент (метрика, учитывающая задержки обработки инцидента на соответствующей линии и отражающая степень соответствия времени обработки инцидента установленному); $K_{2}$ - степень удовлетворенности пользователей качеством и сроком решения инцидентов (доля своевременно закрытых инцидентов с учётом качества их обработки); $P_{\text {отк. }}-$ доступность каналов обработки обрашений (вероятность отказа в обслуживании).

Значения метрики $P_{\text {отк. }}$ рассчитаны для первой линии поддержки, поскольку поток входящих обращений обрабатывается только на ней.

Для расчёта был использован следующий набор статистических данных, собранных на

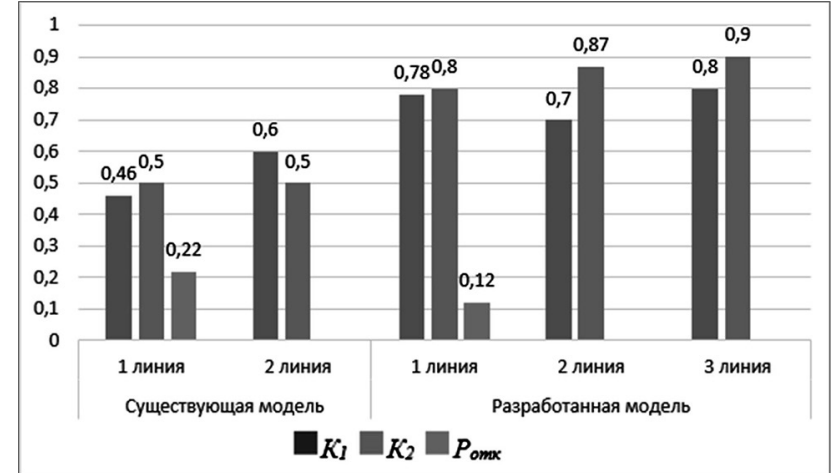

Рис. 3. Сравнительный анализ показателей метрик существующей и разработанной моделей

протяжении определенного периода времени по результатам функционирования системы управления инцидентами ИТ-предприятия: сроки обработки инцидентов первой, второй и третьей линии, время обработки инцидента на первой, второй и третьей линиях, оценка качества консультаций первой линии по $n$-бальной шкале, оценка качества решения инцидента с учетом количества возвратов на доработку, оценка сроков решения инцидента, количество обращений за день/неделю.

Результаты сравнительного анализа существующей и разработанной модели представлены на рис 3.

Скорость решения инцидентов $K_{1}$ на всех трех линиях поддержки в разработанной модели по сравнению с показателями существующей модели возросла на 43,4\%. Степень удовлетворенности пользователей $K_{2}$ выросла на $81 \%$. Доступность каналов обработки $P_{\text {отк }}$ - на $45,4 \%$.

Динамика изменения метрик и оценки степени их приближения к уровням: идеальный, регламентированный и критический представлены на рис 4.

Идеальный уровень (И) определяет значения, к которым метрика должна стремиться. Нормальный (регламентированный) уровень (Н) определяет среднее фактическое значение, соответствующее требованиям, регламентированным в SLA. Критический (недопустимый) уровень (К) свидетельствует о том, что про-

Рис. 4. Изменение показателей скорости решения инщидентов $K_{l}$, степени удовлетворенности пользователей $K_{2}$ и вероятности отказа в обслуживании $P_{\text {отк }}$

в существующей и разработанной моделях

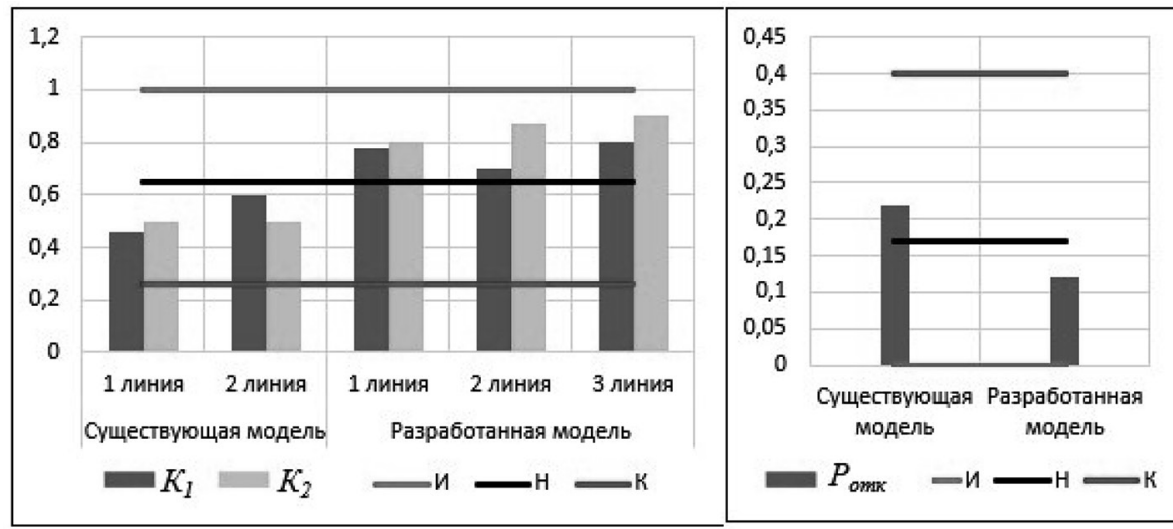

Open education $\diamond$ V. 24. № 3.2020 
цесс управления инцидентами не удовлетворяет установленным требованиям.

Сравнительный анализ показал, что в разработанной модели значения показателей ключевых метрик существенно лучше, чем в существующей модели.

В разработанной модели предполагается возможность использования альтернативных каналов обрашения пользователей, таких, как голосовое сообщение, социальные сети, мессенджеры, формы обратной связи через сайт, либо через мобильное приложение. В существующей модели процесса управления инцидентами использование дополнительных каналов обработки обращений не предусмотрено, а при обращении через такие каналы как телефон и электронная почта - обращение зачастую попадает в очередь, что может быть приравнено к отказу в обслуживании. При обращении через электронную почту возникают ситуации, когда на почтовый сервер поступает достаточно большое количество писем, в результате чего срабатывает спам-фильтр и часть обращений теряется.

В разработанной модели предложено использование единой структурированной базы знаний, которая непрерывно актуализируется, доступ к ней открыт для всех линий поддержки, что позволяет в режиме реального времени обеспечить консультацию пользователя. Единая база знаний обеспечивает повышение уровня компетентности службы технической поддержки, что, в свою очередь, исключает передачу инцидента без предварительной консультации на вторую и третью линии поддержки.

Важное значение имеют сроки и качество разрешения инцидентов, большинство пользователей определяют качество услуги именно сроками устранения. Третья дополнительная линия поддержки обеспечивает решение приоритетных инцидентов в установленные сроки, а также путём мониторинга ошибок и проведения диагностики системы позволяет сделать прогноз причины инцидента и времени его устранения.

\section{Заключение}

Для достижения цели исследования - повышения эффективности процесса управления инцидентами в информационной системе предприятия был проведен анализ существующих подходов к его построению. В результате анализа выявлена типовая модель процесса управления инцидентами, позволившая определить наиболее существенные недостатки (образова- ние очередей на обработку заявок, низкая доступность каналов обработки обращений, не структурированность и неактуальность используемой базы знаний, проблема маршрутизации инцидентов, а именно передача инцидента на 2-й уровень поддержки без его разрешения на 1-м уровне, превышение установленных сроков обслуживания инцидентов и др.).

В соответствии с основополагающим принципами методологии СОВIT и рекомендациями библиотеки ITIL разработана трехуровневая модель обработки инцидентов с использованием единой базы знаний, обеспечивающей поддержание процесса в актуальном состоянии и своевременное реагирование на поступающие запросы. Третья линия поддержки, осуществляет обработку сложных инцидентов, мониторинг ошибок и сбоев, имеющих критический и блокирующий приоритеты.

Предложен подход к обеспечению повышения доступности каналов обработки обращений путем расчета их минимально необходимого числа.

В разработанной модели предложено использовать единую структурированную база знаний, которая актуализируется второй и третьей линиями поддержки. База знаний доступна для всех линий поддержки, этим самым решается проблема маршрутизации инцидентов на вторую линию поддержки без оказания первичной консультации на первой линии. Указанные предложения существенно повышают скорость обработки инцидентов, что положительно сказывается на степени удовлетворенности пользователей сервисом.

Для проведения сравнительного анализа предлагаемой и существующей моделей процесса управления инцидентами предложено использование релевантных метрик, позволяющих объективно оценить степень достижения показателями процесса их целевых значений, т.е. оценить качество управления инцидентами: процессной (скорости решения инцидента), технологической (доступности каналов обработки обращений), и сервисной (степени удовлетворенности пользователей). Обоснованы формальные математические методы количественной оценки указанных метрик.

Сравнительный анализ, проведенный в ходе исследования, показал, что при применении разработанной модели улучшились показатели скорости решения инцидентов на $43,4 \%$, степени удовлетворенности пользователей на $81 \%$ и доступности каналов обработки обращений на $45,4 \%$. 


\section{Литература}

1. Готтшальк Петер, Солли-Сетер Ханс. ИТ-аутсорсинг. Построение взаимовыгодного сотрудничества (Managing Successful IT Outsourcing Relationships). Пер. с англ. А. Петров, А. Сатунин. Издательство: Альпина Паблишер, 2007. 394 с.

2. Уайт Терри. Чего хочет бизнес от IT. Стратегия эффективного сотрудничества руководителей бизнеса и IT-директоров (What Business Really Wants from IT: A Collaborative Guide for Business Directors and CIOs). Пер. с англ. А. Поплавская. Издательство: Гревцов Паблишер, 2007. 256 с.

3. Free ITIL, Best Management Practice, YeSSoft. [Электрон. peсурс]. 2017. 258 с. Режим доступа: http://www.wikiitil.ru/books/2017_Free ITIL.pdf (Дата обрашения: 30.04.2020)

4. ITSM Forum. Словарь терминов и определений ITIL 2011 на русском языке версии 2.0, 29 июля 2011г. на основе английской версии 1.0, 29 июля 2011г [Электрон. ресурс]. Режим доступа: http://www.itsmforum.ru/reference/itilglossary/ (Дата обрашения: 30.04.2020)

5. INTUIT, Национальный открытый университет. ITIL/ITSM - концептуальная основа процессов ИС-службы (курс лекций) [Электрон. pесурс]. Режим доступа: https://www.intuit. $\mathrm{ru} /$ studies/courses/1164/260/lecture/6640? page $=2$ (Дата обращения: 30.04.2020)

6. Иванов Д.Б. Разработка системы управления функционированием службы технической поддержки Интернет-провайдера на базе библиотеки ITIL: диссертация кандидата технических наук. Воронеж, 2008. 142 с.

7. Тушавин, В. А. Модели и методы управления качеством разрешения инцидентов при реализации информационно-коммуникационных услуг: диссертация кандидата технических наук. Санкт-Петербург, 2012. 175 с.

8. Шпер А. В. Сервисный менеджмент в информационных технологиях: диссертация кандидата экономических наук. Москва, 2007. 212 с.

9. Максимов Н.В., Партыка Т.Л., Попов Т.Л. Современные информационные технологии. М.: Форум, 2012 г. 512 с.

\section{References}

1. Gottshal'k Peter, Solli-Seter Khans. ITautsorsing. Postroyeniye vzaimovygodnogo sotrudnichestva (Managing Successful IT Outsourcing Relationships). Per. s angl. A. Petrov, A. Satunin = IT outsourcing. Building a mutually beneficial collaboration (Managing Successful IT Outsourcing Relationships). Tr. from Eng. A. Petrov, A. Satunin. Publisher: Alpina Publisher; 2007. 394 p. (In Russ.)

2. Uayt Terri. Chego khochet biznes ot IT. Strategiya effektivnogo sotrudnichestva rukovoditeley biznesa i IT-direktorov (What Business Really Wants from IT: A Collaborative Guide for Business Directors and CIOs). Per. s angl. A. Poplavskaya $=$ What the business wants from IT. An effective collaboration strategy for business leaders and IT directors (What Business Really Wants from IT: A Collaborative Guide for Business Directors and
10. Тейлор Ш. Создание услуг высокого качества и управление ими. М.: itSMF России, 2012. $64 \mathrm{c}$.

11. Брукс Питер. Метрики для управления ИТ-услугами (Metrics for IT Service Management). Серия: Библиотека IBS, пер. с англ. В. Первушина. М.: Издательство «Альпина Паблишер», 2008. 288 с.

12. Журавлев Р. Иллюстрированный ITSM. М.: Лайвбук, 2013. 125 с.

13. Томпсон А.А., Стрикленд А. Дж. Стратегический менеджмент: концепции и ситуации для анализа, 12-е издание, пер. с англ. М.: Издательский дом «Вильямс», 2002.

14. Ингланд Роб. Введение в реальный ITSM (Introduction to Real ITSM). Пер. с англ. P. Журавлев. М.: Гаятри/Livebook, 2010. 132 с.

15. Ян В.Б. (Jan van Bon). ИТ Сервис-менеджмент. Вводный курс на основе ITIL. Издатель: Van Haren Publishing, по заказу ITSMF Netherlands. 303 c.

16. Ян Ван Бон, Пондман Д. ИТ Сервис-менеджмент, введение. Перевод на русский язык под редакцией М.Ю. Потоцкого. М.: Открытые Системы, 2003.

17. Брукс Питер. Метрики для управления ИТ-услугами (Metrics for IT Service Management). Серия: Библиотека IBS, пер. с англ. В. Первушина. М.: Издательство «Альпина Паблишер», 2008. $288 \mathrm{c}$

18. Inframanager. Управление инцидентами и проблемами - понятия и принципь [Электрон. ресурс]. Инфраменеджер. Режим доступа: https://www.inframanager.ru/library/ about-methodology/upravlenie-incidentami/ (Дата обрашения: 30.04.2020)

19. Исайченко Д., Журавлёв Р. ITSM. Руководство по измерению. М.: Лайвбук, 2015. $141 \mathrm{c}$.

20. Статьи консультантов Cleverics. Измерение процесса управления инцидентами и запросами пользователей [Электрон. ресурс]. Режим доступа: https://cleverics.ru/subject-field/ articles/589-incident-management-measurement. (Дата обращения:30.04.2020).

CIOs). Tr. from Eng. A. Poplavskaya. Publisher: Grevtsov Publisher; 2007. 256 p. (In Russ.)

3. Free ITIL, Best Management Practice, YeSSoft. [Internet]. 2017. 258 p. Available from: http://www.wikiitil.ru/books/2017 Free ITIL.pdf (cited 30.04.2020)

4. ITSM Forum. Slovar' terminov i opredeleniy ITIL 2011 na russkom yazyke versii 2.0, 29 iyulya 2011g. na osnove angliyskoy versii 1.0, 29 iyulya $2011 \mathrm{~g}=$ ITSM Forum. Glossary of ITIL 2011 terms and definitions in Russian version 2.0, July 29, 2011 based on the English version 1.0, July 29, 2011 [Internet]. Available from: http://www.itsmforum.ru/ reference/itil-glossary/ (cited 30.04.2020) (In Russ.)

5. INTUIT, Natsional'nyy otkrytyy universitet. ITIL/ITSM - kontseptual'naya osnova protsessov IS-sluzhby (kurs lektsiy) = INTUIT, National Open University. ITIL / ITSM - the conceptual basis of IP service processes (lecture course) [Internet]. 
Available from: https://www.intuit.ru/studies/ courses $/ 1164 / 260 /$ lecture $/ 6640$ ? page $=2 \quad$ (cited 30.04.2020). (In Russ.)

6. Ivanov D.B. Razrabotka sistemy upravleniya funktsionirovaniyem sluzhby tekhnicheskoy podderzhki Internet-provaydera na baze biblioteki ITIL: dissertatsiya kandidata tekhnicheskikh nauk $=$ Development of a control system for the functioning of the technical support service of the Internet provider based on the ITIL library: the dissertation of the candidate of technical sciences. Voronezh, 2008. 142 p. (In Russ.)

7. Tushavin, V. A. Modeli i metody upravleniya kachestvom razresheniya intsidentov pri realizatsii informatsionno-kommunikatsionnykh uslug: dissertatsiya kandidata tekhnicheskikh nauk = Models and methods of quality management of incident resolution in the implementation of information and communication services: the dissertation of the candidate of technical sciences. St. Petersburg, 2012. 175 p. (In Russ.)

8. Shper A. V. Servisnyy menedzhment V informatsionnykh tekhnologiyakh: dissertatsiya kandidata ekonomicheskikh nauk $=$ Service management in information technology: the dissertation of the candidate of economic sciences. Moscow, 2007. 212 p. (In Russ.)

9. Maksimov N.V., Partyka T.L., Popov T.L. Sovremennyye informatsionnyye tekhnologii = Modern information technologies. Moscow: Forum, 2012. 512 p. (In Russ.)

10. Teylor SH. Sozdaniye uslug vysokogo kachestva $\mathrm{i}$ upravleniye $\mathrm{imi}=$ Creating and managing high quality services. Moscow: itSMF Russia; 2012. 64 p. (In Russ.)

11. Bruks Piter. Metriki dlya upravleniya ITuslugami (Metrics for IT Service Management). Seriya: Biblioteka IBS, per. s angl. V. Pervushina = Metrics for IT Service Management. Series: IBS Library. Tr. from Eng. V. Pervushina. Moscow: Alpina Publisher Publishing House; 2008. 288 p. (In Russ.)

12. Zhuravlev R. Illyustrirovannyy ITSM = Illustrated ITSM. Moscow: Livebook; 2013. 125 p. (In Russ.)

13. Tompson A.A., Striklend A. Dzh.
Strategicheskiy menedzhment: kontseptsii i situatsii dlya analiza, 12-ye izdaniye, per. s angl = Strategic Management: Concepts and Situations for Analysis, 12th edition. TR. from Eng. Moscow: Williams Publishing House; 2002. (In Russ.)

14. Ingland Rob. Vvedeniye $\mathrm{V}$ real'nyy ITSM (Introduction to Real ITSM). Per. sangl. R. Zhuravlev $=$ Introduction to Real ITSM (Introduction to Real ITSM). Tr. from Eng. R. Zhuravlev. Moscow: Gayatri/Livebook; 2010. 132 p. (In Russ.)

15. Yan V. B. (Jan van Bon). IT Servis menedzhment. Vvodnyy kurs na osnove ITIL = IT Service Management. Introductory course based on ITIL Publisher: Van Haren Publishing, commissioned by ITSMF Netherlands. 303 p. (In Russ.)

16. Yan Van Bon, Pondman D. IT Servismenedzhment, vvedeniye. Perevod na russkiy yazyk pod redaktsiyey M.YU. Pototskogo = IT Service Management, Introduction. Translation into Russian edited by M.Yu. Potocki. Moscow: Open Systems; 2003. (In Russ.)

17. Bruks Piter. Metriki dlya upravleniya ITuslugami (Metrics for IT Service Management). Seriya: Biblioteka IBS, per. s angl. V. Pervushina = Metrics for IT Service Management. Series: IBS Library. Tr. from Eng. V. Pervushina. Moscow: Alpina Publisher Publishing House; 2008. 288 p. (In Russ.)

18. Inframanager. Upravleniye intsidentami i problemami - ponyatiya $\mathrm{i}$ printsipy $=$ Incident and problem management - concepts and principles [Internet]. Infrastructure manager. Available from: https://www.inframanager.ru/library/aboutmethodology/upravlenie-incidentami/ (cited 30.04.2020). (In Russ.)

19. Isaychenko D., Zhuravlov R. ITSM. Rukovodstvo po izmereniyu $=$ ITSM. Measurement Guide. Moscow: Livebook: 2015. 141 p.

20. Stat'i konsul'tantov Cleverics. Izmereniye protsessa upravleniya intsidentami i zaprosami pol'zovateley $=$ Articles consultants Cleverics. Measuring the process of managing incidents and user requests [Internet]. Available from: https://cleverics. $\mathrm{ru} /$ subject-field/articles/589-incident-managementmeasurement. (cited30.04.2020). (In Russ.)

\section{Сведения об авторах}

\section{Андрей Александрович Микрюков к.т.н., доцент кафедры Прикладной информатики и информационной безопасности Российский экономический университет им. Г.В. Плеханова, \\ Москва, Россия \\ Эл.noчma: mikrukov.aa@rea.ru}

\section{Алексина Владимировна Куулар}

старший спещиалист АО «ЦПЛ», аспирант кафедры Прикладной информатики и информационной безопасности Российский экономический университет им. Г.В. Плеханова,

Москва, Россия

Эл.noчma: kuularalexa@mail.ru

\section{Information about the authors \\ Andrei A. Mikryukov}

Ph.D., Associate Professor of the Department of Applied Informatics and Information Security Russian University of Economics named G.V. Plekhanova

Moscow, Russia

E-mail:mikrukov.aa@rea.ru

\section{Aleksina Vl. Kuular}

Senior Specialist Joint-Stock Company «Loyalty Program Center», graduate student of the Department of Applied Informatics and Information Security Russian University of Economics named G.V. Plekhanova

Moscow, Russia

E-mail: kuularalexa@mail.ru 